\title{
Heartbeat: Text messaging to improve health
}

It has been challenging to develop effective interventions to increase the number of people engaged in heart healthy behaviours. We all look at our phones all the time; text messaging offers a simple way to reach our patients one on one. Burn and colleagues ${ }^{1}$ used a Markov model, based on a previous randomised trial and meta-analysis, to evaluate the effect of a simple text messaging programme for secondary prevention of adverse cardiovascular disease (CVD) events and improvement in quality adjusted life years (QALYs) in patients with known coronary artery disease (CAD). The programme, called TEXT ME, consists of 4 weekly text messages for 6 months with message content targeted to each participant's baseline cardiac risk factor profile across the domains of general health and medication adherence, diet, exercise, and smoking cessation. (figure 1). Overall, the TEXT ME programme was estimated to result in 563 fewer myocardial infarctions, 361 fewer strokes and 1143 additional QALYs in a target population of 50 thousand CAD patients. Cost savings would be substantial, given that the text messaging programme is quite inexpensive.

In the accompanying editorial, Marcolino and colleagues note that 'It is also important to emphasise that it was an educational tool and an effective way of empowering patients to make informed decisions.' ${ }^{2}$ They go on to suggest that 'The high effectiveness and low cost of this intervention, however, turn it as an excellent candidate to be scaled to reach a global population. There is a potential of even greater benefit in low-income and middle-income countries, where the heaviest burden of cardiovascular disease reside and where the access of rehabilitation programmes and specialised care may be impaired.'

In addition to interventions aimed at all CAD patients, more costly interventions can be directed to those who will benefit most if we can achieve adequate risk stratification. Poppe and colleagues, ${ }^{3}$ developed

Division of Cardiology, University of Washington, Seattle, Washington, USA

Correspondence to Professor Catherine M Otto, Division of Cardiology, University of Washington, Seattle, Washington 98105, USA; cmotto@uw.edu

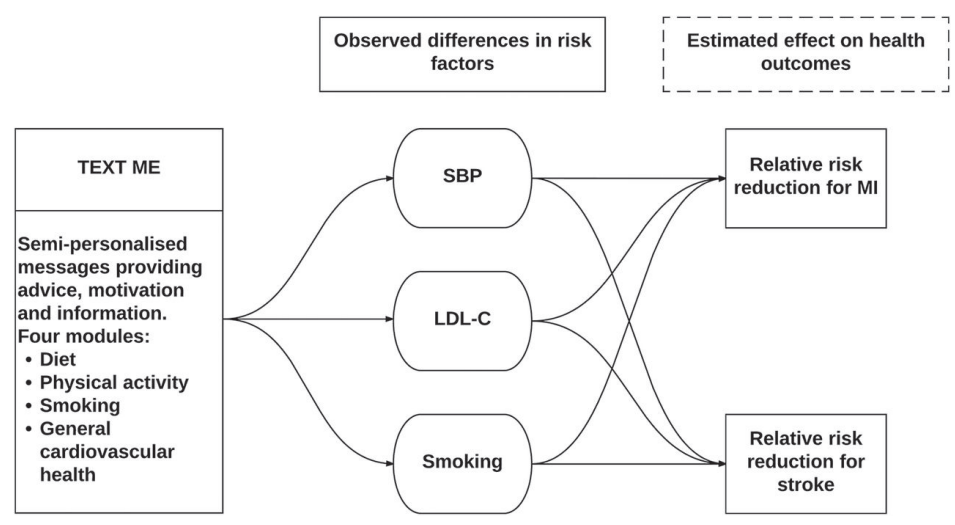

Figure 1 Estimated effect of TEXT ME on health outcomes. Differences in risk factors were observed at 6 months. Relative risk reductions associated with changes in these risk factors were estimated and extrapolated into the future. ${ }^{1}$ LDL-C, low-density lipoprotein cholesterol; MI, myocardial infarction; SBP, systolic blood pressure; TEXT ME, Tobacco, Exercise and Diet Messages.

a CVD risk score, called PREDICT-CVD, based on almost 25 thousand patients under age 80 years with known CVD in a New Zealand primary care cohort. In addition to conventional risk factors, the risk score included ethnicity, comorbidities, body mass index, and renal function. Predicted event rates matched actual event rates in the original cohort with overestimation of risk in those at highest risk in a second cohort. (figure 2).

Banerjee $^{4}$ comments that although we have robust tools for identifying people at risk of incident CVD, we have only limited ability to predict recurrent CVD events in patients with known CAD. Risk stratification could allow more intensive monitoring of secondary prevention in those at highest risk; thus PREDICT-CVD has great value, particularly because it is

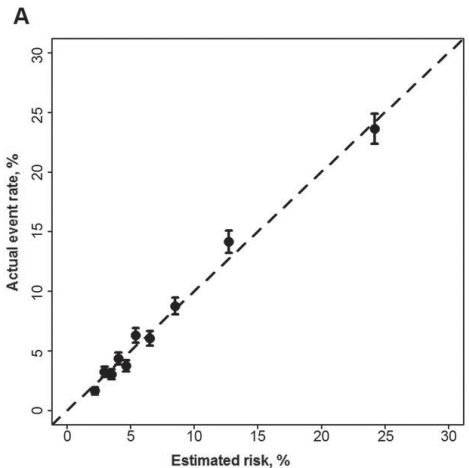

based on routine clinical data. Still, there are several issues to address in future studies. Most CVD risk scores predict only atherosclerotic events; other types of heart disease such as heart failure and atrial fibrillation are increasingly common. We also need to show that risk stratification improves clinical outcomes, not just personalization of secondary prevention therapies. 'Finally, at present, the potential of Electronic health records (EHR) for development and validation of risk prediction tools across diseases remains largely untapped with few multicentre studies and even fewer with validation across sites.' 'The current paradigm of multivariable Cox regression models informed by prospective cohort studies testing the incremental gain of novel biomarkers one by one needs to be challenged.'

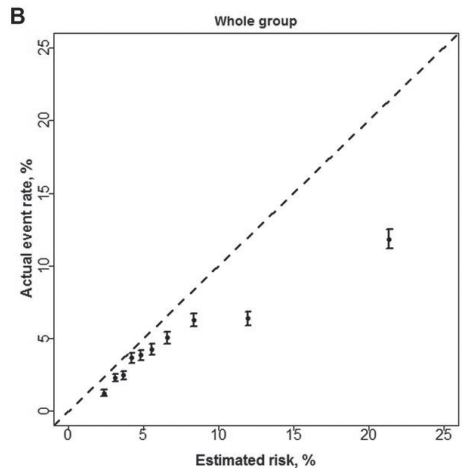

Figure 2 Model calibration in the development and external validation cohorts. Calibration of the risk equation in $(A)$ the development cohort (New Zealand) and (B) the external cohort (UK). For both plots, calibration is shown as the actual survival from Kaplan-Meier analysis against estimated risk (in deciles). ${ }^{3}$ Dashed line=perfect calibration. 


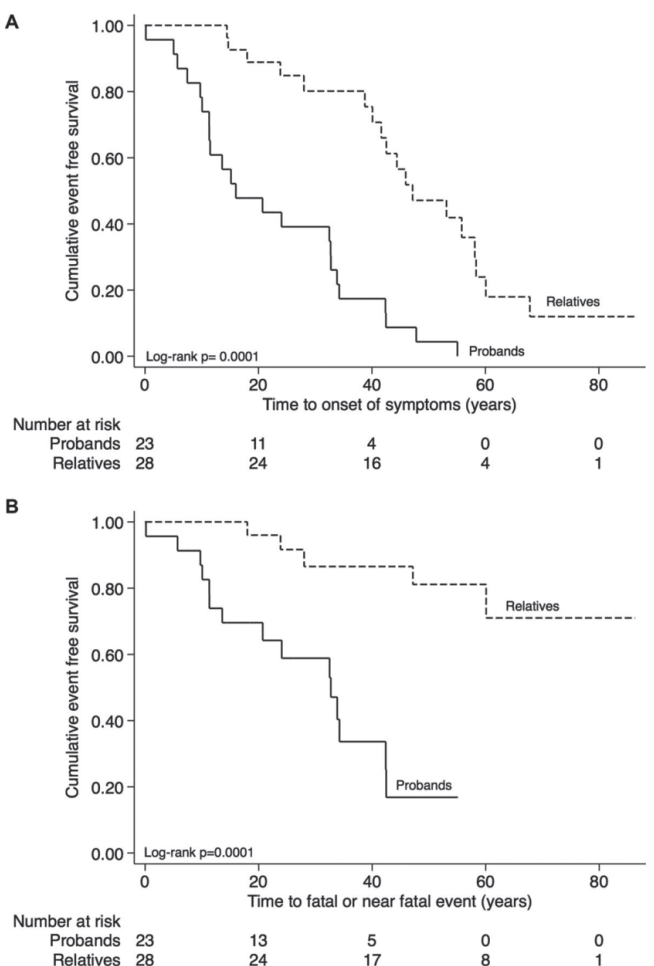

Figure 3 (A) Kaplan-Meier plot presenting time (years) from birth to onset of symptoms. (B) Kaplan-Meier plot presenting time (years) from birth to fatal or near-fatal event (sudden cardiac death (SCD), aborted SCD). ${ }^{5}$ Probands $=23$, relatives $=28$.

Catecholaminergic polymorphic ventricular tachycardia (CPVT) is a rare condition that presents with sudden death in about $1 / 3$ of affected patients. However, as the study by Broendberg and colleagues ${ }^{5}$ shows, the dominantly inherited ryanodine receptor-2 (RyR2) gene has variable penetrance with gene-positive family members having fewer symptoms and a lower risk of sudden cardiac death compared with proband patients presenting with symptoms or sudden death (figure 3).
As Pflaumer ${ }^{6}$ explains in an editorial, the RyR2 gene is important in regulation of cardiac myocyte calcium homeostasis; thus, anti-arrhythmic medications may be effective in some patients. Indications for an implanted cardiac defibrillator are unclear, with a high rate of device complications in these patients. He concludes that 'this study underlines that one should not underestimate CPVT, as patients can be asymptomatic for a long period in their life and then present with a fatal or a near-fatal event. Family screening and a

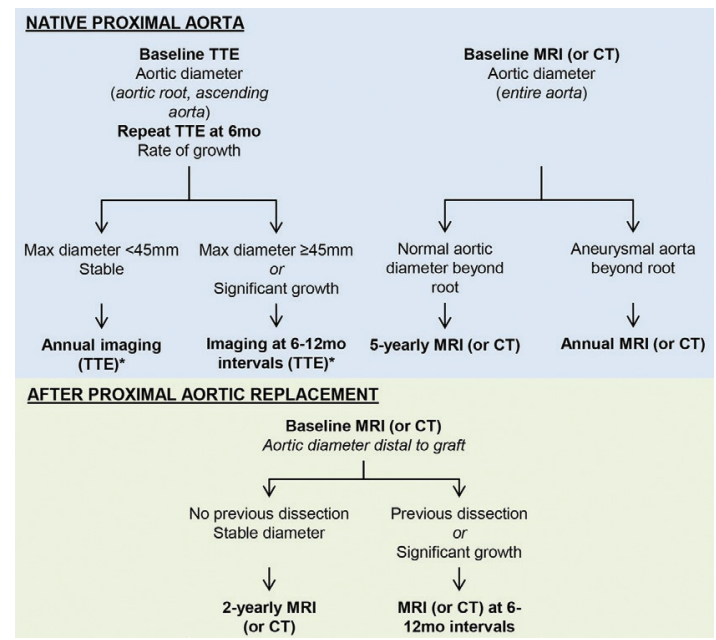

Figure 4 Imaging surveillance of the aorta in patients with Marfan syndrome. * If visualisation of aortic root and ascending aorta is inadequate, MRI (or CT) should be employed. ${ }^{7}$ mo, months; SoV, sinuses of Valsalva; STJ, sinotubular junction; TTE, transthoracic echocardiography.

very thorough diagnostic approach with multiple exercise tests can be life-saving.' However, 'we should also ask ourselves if an arrest in an undiagnosed, untreated CPVT patient should always trigger an ICD implantation, although this might be walking a bit closer towards the abyss.'

The Education in Heart article in this issue, ${ }^{7}$ provides a concise summary of the pathophysiology and genetics of inherited causes of aortic dilation. (figure 4). A practical clinical approach is provided with tables summarizing genotype-phenotype correlates and simple flow charts for patient management.

A state-of-the art review article provides an overview of the prevalence and causes of atherosclerosis in patients with congenital heart disease. ${ }^{8}$ There are two Image Challenge questions to test your diagnostic skills, one of which was very popular on Twitter. $^{9} 10$

Competing interests None declared.

Provenance and peer review Commissioned; internally peer reviewed.

(c) Article author(s) (or their employer(s) unless otherwise stated in the text of the article) 2017. All rights reserved. No commercial use is permitted unless otherwise expressly granted.

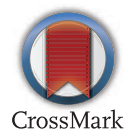

To cite Otto CM. Heart 2017;103:887-888.

Heart 2017;103:887-888.

doi:10.1136/heartjnl-2017-311784

\section{REFERENCES}

1 Burn E, Nghiem S, Jan S, et al. Cost-effectiveness of a text-message program for cardiovascular disease secondary prevention. Heart 2017;103:923-30.

2 Marcolino MS, Oliveira JA, Ribeiro AL. Text messaging for prevention and treatment of cardiovascular disease: time to move on. Heart 2017;103:893-4.

3 Poppe KK, Doughty RN, Wells S, et al. Developing and validating a cardiovascular risk score for patients in the community with prior cardiovascular disease. Heart 2017;103:917-22.

4 Banerjee A. Predicting the future of cardiovascular risk prediction. Heart 2017;103:891-2.

5 Broendberg AK, Nielsen JC, Bjerre J, et al. Nationwide experience of catecholaminergic polymorphic ventricular tachycardia caused by RyR2 mutations. Heart 2017;103:901-9.

6 Pflaumer A. Catecholaminergic polymorphic tachycardia: underestimated and overtreated? Heart 2017;103:889-90.

7 Kuijpers JM, Mulder BJ. Aortopathies in adult congenital heart disease: management strategies and indications for surgery. Heart 2017;103:952-65.

8 Tarp JB, Jensen AS, Engstrøm T, et al. Cyanotic congenital heart disease and atherosclerosis. Heart 2017; 103:897-900.

9 Moss AJ, Lim KH, Japp AG. A 49-year-old with chest pain and collapse. Heart 2017;103:930.

10 William W, Ring L, Salahshouri P. 84 Year Old female with dizziness. Heart 2017;103:966. 